Regular Article

\title{
Cyclodextrin-functionalized mesostructured silica nanoparticles for removal of polycyclic aromatic hydrocarbons
}

\author{
Fuat Topuz ${ }^{\mathrm{a}, *}$, Tamer Uyar $^{\mathrm{a}, \mathrm{b}, *}$ \\ ${ }^{a}$ UNAM-National Nanotechnology Research Center, Bilkent University, 06800 Ankara, Turkey \\ ${ }^{\mathrm{b}}$ Institute of Materials Science E' Nanotechnology, Bilkent University, 06800 Ankara, Turkey
}

\section{G R A P H I C A L A B S T R A C T}
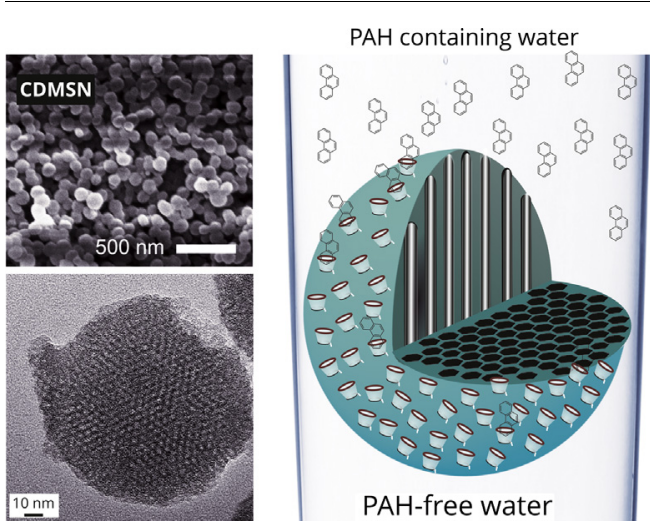

\section{A R T I C L E I N F O}

\section{Article history:}

Received 26 December 2016

Revised 21 February 2017

Accepted 2 March 2017

Available online 4 March 2017

\section{Keywords:}

Mesostructured silica nanoparticles (MSN)

Polycyclic aromatic hydrocarbons (PAH)

Cyclodextrin

Water treatment

\begin{abstract}
A B S T R A C T
Polycyclic aromatic hydrocarbons (PAHs) are the byproducts of the incomplete combustion of carbonbased fuels, and have high affinity towards DNA strands, ultimately exerting their carcinogenic effects. They are ubiquitous environmental contaminants, and can accumulate on tissues due to their lipophilic nature. In this article, we describe a novel concept for PAH removal from aqueous solutions using cyclodextrin-functionalized mesostructured silica nanoparticles (CDMSNs) and pristine mesostructured silica nanoparticles (MSNs). The adsorption applications of MSNs are greatly restricted due to the absence of surface functional groups on such particles. In this regard, cyclodextrins can serve as ideal functional molecules with their toroidal, cone-type structure, capable of inclusion-complex formation with many hydrophobic molecules, including genotoxic PAHs. The CDMSNs were synthesized by the surfactanttemplated, $\mathrm{NaOH}$-catalyzed condensation reactions of tetraethyl orthosilicate (TEOS) in the presence of two different types of cyclodextrin (i.e. hydroxypropyl- $\beta$-cyclodextrin (HP- $\beta$-CD) and native $\beta$-cyclodextrin ( $\beta$-CD)). The physical incorporation of CD moieties was supported by XPS, FT-IR, NMR, TGA and solid-state ${ }^{13} \mathrm{C}$ NMR. The CDMSNs were treated with aqueous solutions of five different PAHs (e.g. pyrene, anthracene, phenanthrene, fluorene and fluoranthene). The functionalization of MSNs with cyclodextrin moieties significantly boosted the sorption capacity $(q)$ of the MSNs up to $\sim 2$-fold, and the $q$ ranged between 0.3 and $1.65 \mathrm{mg}$ per gram CDMSNs, of which the performance was comparable to that of the activated carbon.
\end{abstract}

(c) 2017 Elsevier Inc. All rights reserved.

\footnotetext{
* Corresponding authors at: UNAM-National Nanotechnology Research Center, Bilkent University, 06800 Ankara, Turkey (T. Uyar and F. Topuz).

E-mail addresses: fuat.topuz@rwth-aachen.de (F. Topuz), tamer@unam.bilkent.edu.tr (T. Uyar).
} 


\section{Introduction}

Today's world faces an intriguing contamination problem of water resources, soils and atmosphere with many types of pollutants, including polycyclic aromatic hydrocarbons (PAHs). PAHs constitute a large chemical family of fused benzene rings with their potent carcinogenic, teratogenic and mutagenic activities [1,2]. On contact with body tissues, they undergo metabolic activation to reactive epoxide compounds through an enzymatic oxidation pathway initiated by cytochrome P450 (CYP), and the formed reactive metabolites are capable of binding to nucleotides on a cellular DNA, forming PAH-DNA adducts. If not repaired, these adducts may induce mutations in oncogenes and suppression genes, eventually leading to the transformation of a normal cell into a cancer cell [3-5]. In vivo studies with mice demonstrated mutagenicity of PAHs; for example, pregnant mice exposed to benzo( $\alpha$ )pyrene (B $[\alpha] p)$ for 10 days showed the effects of birth defects [6,7]. Similarly, mice exposed to $923 \mathrm{ppm}$ of $\mathrm{B}[\alpha] \mathrm{p}$ in food for months displayed liver problems [8]. In today's world, the presence of PAHs in the environment is indeed very common, extremely serious, and moreover, $\mathrm{PAH}$ level rises with proximity to urban centers [9-12]. Humans are continuously exposed to some levels of PAHs through many different sources, including smoking, diet and drinking water. Since the PAHs are ubiquitously present in water sources, and have a lipophilic nature, they can easily accumulate on tissues, and exert its long-term effects in vivo. Despite being hydrophobic in nature, PAHs are water soluble to a certain extent, and have been found in ground water (1.0-10.0 ng/L), rain water (2.7-7.3 ng/L), tap water (2.5-9.0 ng/L) and surface water (10-830 ng/L) [13]. Further, the total potential dose of carcinogenic PAHs for a nonsmoker adult has been estimated to be a median $3 \mu \mathrm{g} / \mathrm{day}$, while sometimes reaching to a maximum value of $15 \mu$ g per day [13]. One important source of this uptake is polluted drinking water sources, particularly for developing countries, where they are often hampered by the lack of limited enforcement of water quality standards and available technologies for effective water-remediation. Their consistent exposure is associated with diseases (e.g. asthma and cardiovascular problems) in addition to many types of cancers. The most common consequence of PAH exposure is p53 mutations in lung cancers due to DNA damage caused by cigarette smoke carcinogens, including PAHs [14]. Thus, several attempts have been made in recent years for the efficient removal of trace PAHs from contaminated soils, water and atmosphere using functional material platforms, including a semi-rigid cyclophane (ExBox) [15], cyclodextrins [16], calix[n]arenes [17], and metal-ligand diazapyrenium-based metallocycles [18], and more recently, photo-switchable nanoparticles that aggregate upon irradiation [19].

Mesoporous silica nanoparticles (MSNs) are chemically and thermally stable nanomaterials having pores in the range of $2-50 \mathrm{~nm}$. They can offer a very large surface area $\left(\sim 800 \mathrm{~m}^{2} / \mathrm{g}\right)$, and thus have taken a considerable interest in many diverse fields of science, industry and technology [20-23]. Those nanomaterials are synthesized by the condensation reactions of tetraethyl orthosilicate (TEOS) in the presence of a surfactant (e.g. cetyltrimethylammonium bromide (CTAB)) as a pore-templating agent under acidic or basic catalysis. The reactions are initiated with the hydrolysis of alkoxy groups into silanols, and followed by condensation reactions, ultimately yielding silica particles. The internal porous structure of such particles allows the transport of small (bio)molecules (e.g. drugs, proteins, peptides and oligos) and releases payloads into targeted areas by intra- or extracellularly after having decorated with bioactive ligands. Quite recently, it was shown that they can even bypass the plant cell wall with plasmid modification, and subsequently release the embedded drugs into the cytosol [24]. In addition to these applications, such nanoplatforms have been employed as sorbent materials for water remediation, or the separation of molecules based on the molecular size, hydrophobic/hydrophilic balance and ionic charge [25-27]. The structural characteristics of MSNs (e.g. shape, size and pore architecture) can be tailored by means of a suitable synthesis route. Further, these nanomaterials can be modified by either physical or chemical routes with a variety of functional motifs, including cyclodextrins.

Cyclodextrins are cyclic oligosaccharides, capable of forming host-guest complexes with small lipophilic molecules using their hydrophobic toroidal cavity, of which the exterior is hydrophilic because of the presence of the primary and secondary hydroxyl groups. In an aqueous medium, those CD motifs can complex with a wide range of small hydrophobic compounds, including PAHs, fragrances and other types of lipophilic molecules, and the complexation notably enhances water solubility of guest hydrophobic compounds $[28,29]$. Hence, they have been heavily used in pharmacy, agriculture, textile, catalysis, hygiene, cosmetic and food industry [30-33]. Further, in our recent studies we have shown that electrospun nanofibers can be functionalized with cyclodextrins as molecular filters for water filtration [34,35] removal of volatile organic compounds (VOCs) [36-40] including aniline, benzene, styrene and toluene, and the removal of phenanthrene from an aqueous system $[41,42]$.

Given that a highly-ordered honeycomb-like meso- or microporous structure with a large surface area, cyclodextrinfunctionalized MSNs (CDMSNs) would be an interesting challenge for PAH removal from aquatic environments. In such a case, the separation of nanoparticles can be accomplished via centrifugation or filtration. Quite recently, silica aggregates with physically adsorbed graphene sheets were used for the removal of phenanthrene from water [43]. They observed that graphene-decorated silica particles could scavenge phenanthrene in the range of $0.014-1.74 \mathrm{mg}$ per gram particles with an increasing content of graphene. Similarly, Laveille et al. (2010) reported hemoglobin ( $\mathrm{Hb}$ ) functionalized mesoporous silica particles for PAH scavenging [44]. Hemoglobin $\mathrm{Hb}$ ) was physically encapsulated in the pores of commercially available micro-sized silica particles (LiChrospher 60) with a mean pore size of $6 \mathrm{~nm}$ and the particle size of $\sim 10 \mu \mathrm{m}$. These particles were implemented for the covalent attachment of PAHs to Hb over $\mathrm{a}_{2} \mathrm{O}_{2}$-catalyzed reaction. Another study used silica microparticles for the removal of acenaphthene from water with a sorption capacity of $0.7 \mathrm{mg}$ per gram silicagel [45].

In this study, we describe an efficient method for the removal of several PAH compounds from aqueous solutions using physically CD-functionalized MSNs (hpCDMSN and nCDMSN), along with pristine MSNs as a control material (Scheme 1). The CDMSNs were synthesized by the surfactant-templated, base-catalyzed condensation reactions of tetraethyl orthosilicate (TEOS) in the presence of hydroxypropyl- $\beta$-cyclodextrin or native $\beta$-cyclodextrin. Several characterization techniques (e.g. XPS, FT-IR, NMR, TGA and solidstate ${ }^{13} \mathrm{C}$ NMR) were employed to elucidate the structural properties of the nanoparticles. The CDMSNs and pristine MSNs were later used for the removal of five different $\mathrm{PAH}$ compounds (i.e. pyrene, anthracene, phenanthrene, fluorene and fluoranthene) from aqueous solutions. The sorption capacities were determined via fluorescence measurements over changes in the mean fluorescence intensity of the supernatant before and after treatments with the CDMSNs.

\section{Experimental section}

\subsection{Materials}

Polycyclic aromatic hydrocarbons (PAHs) (i.e. pyrene, anthracene, phenanthrene, fluorene and fluoranthene) and tetraethyl orthosilicate (TEOS, 98\%) were purchased from Sigma-Aldrich. 


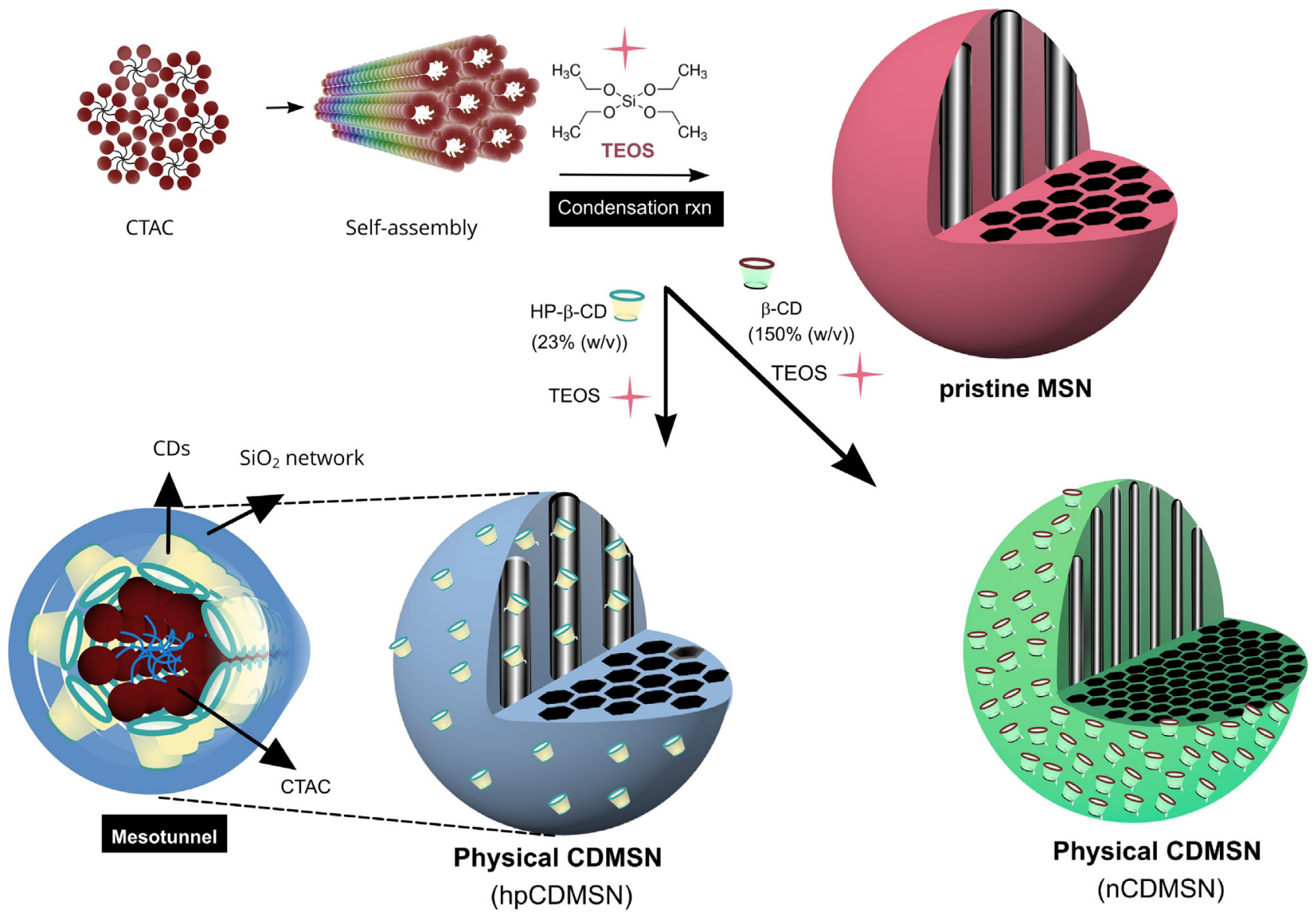

Scheme 1. Synthesis scheme of pristine MSN and physically cyclodextrin-functionalized MSN. The hpCDMSN stands for hydroxypropyl- $\beta$-cyclodextrin functionalized MSN, and nCDMSN denotes to native $\beta$-cyclodextrin functionalized MSN.

Cetyltrimethylammonium chloride (CTAC, >98\%) was received from TCI-Chemicals. Hydroxypropyl- $\beta$-cyclodextrin (HP- $\beta-C D$, CAVASOL-W7 HP) and $\beta$-cyclodextrin ( $\beta$-CD, CAVAMAX-W7) were kindly donated by Wacker Chemie AG. Sodium hydroxide $(\mathrm{NaOH})$ was purchased from Merck.

\subsection{Synthesis of Cyclodextrin-Functionalized Mesostructured Silica Nanoparticles (CDMSNs) and pristine MSNs}

CTAC (0.4 g) was dissolved in water $(96 \mathrm{~mL})$, and the mixture was continuously stirred at $80^{\circ} \mathrm{C}$ for $30 \mathrm{~min}$ until a transparent solution was formed. Afterward, HP- $\beta$-CD ( $23 \%$ (w/v) of TEOS) or $\beta-\mathrm{CD}(150 \%(\mathrm{w} / \mathrm{v})$ of TEOS) was added to the solution for the synthesis of CDMSNs, and followed by the addition of $2 \mathrm{~N} \mathrm{NaOH}$ $(0.7 \mathrm{~mL})$. The condensation reactions were initiated with TEOS by a dropwise addition over several minutes. This synthesis pathway led to the CDMSNs, where cyclodextrin moieties were physically incorporated. These particles were named as hpCDMSN or nCDMSN depending on the type of $C D$ used. For the synthesis of the pristine MSNs, TEOS was directly added to the solution. The nanoparticles were washed with a mixed solvent of acetic acid and methanol (1:3) to remove weakly adsorbed CD and CTAC molecules from the nanoparticles.

\subsection{Characterizations}

2.3.1. Dynamic Light Scattering (DLS) E zeta-potential measurements The particle size was measured by a photon correlation spectroscopy "Malvern Nano ZS ZEN3600 (Malvern Instruments, USA)" at a scattering angle of $173^{\circ}$. Disposable polystyrene cuvettes were used for size measurements in water. "Expert System" software was used for data interpretation. The presented data are average values of three measurements. The dynamic light scattering (DLS) measurements give a Z-average (or cumulant mean) value, which is an intensity mean and the polydispersity index (PDI). The cumulant analysis has the following form;

$\ln \left(g^{(1)}(t)\right)=-\Gamma t+\mu_{2} t^{2}+\cdots$

where $g^{(1)}$ is the first order correlation function, $\bar{\Gamma}$ is the average decay rate and first cumulant, and $\mu_{2}$ is the second cumulant. The value $\mu_{2} / \bar{\Gamma}^{2}$ is known as PDI.

The zeta-potential measurements were measured in the $\mathrm{pH}$ range of 3-12, and an autotitrator (MPT-2, Malvern Instruments) was used to adjust the sample $\mathrm{pH}$.

\subsubsection{Wide-Angle X-ray Diffraction (WAXD) analysis}

WAXD experiments were performed using a PANalytical X'Pert Pro MPD, which was powered by a Philips PW3040/60 X-ray generator and fitted with an X'Celerator detector. Diffraction data was acquired by exposing powder samples to $\mathrm{Cu} \mathrm{K}$-alfa X-ray radiation. $\mathrm{X}$-rays were generated from a $\mathrm{Cu}$ anode supplied with $40 \mathrm{kV}$ and the current of $40 \mathrm{~mA}$. The data were collected over the range of $2-40^{\circ}$ of $2 \theta$. All scans were carried out in 'continuous' mode, and the data were later analyzed by X'Pert Highscore Plus software (version 2.0). 


\subsubsection{Fourier Transform-Infrared (FT-IR) spectroscopy}

FT-IR spectra of the nanoparticles were recorded on a BrukerVERTEX 70 spectrometer. The spectra were taken at a resolution $4 \mathrm{~cm}^{-1}$ after 128 scans accumulation for an acceptable signal/noise ratio.

\subsubsection{X-ray Photoelectron Spectroscopy (XPS)}

The XPS spectra of the nanoparticles were recorded by a X-ray photoelectron spectrometer (Thermo Fisher Scientific, U.K.). As a $\mathrm{X}$-ray source, Al K-alpha X-ray monochromator ( $0.1 \mathrm{eV}$ step size, $12 \mathrm{kV}, 2.5 \mathrm{~mA}$, spot size $400 \mu \mathrm{m}$ ) was used at an electron take-off angle of $90^{\circ}$ from the surface. For each sample, survey spectrum was taken 5 times with $50 \mathrm{~ms}$ dwell time (pass energy $200 \mathrm{eV}$ ). C1 s spectra were taken 10 times with $50 \mathrm{~ms}$ dwell time (pass energy $30 \mathrm{eV}$ ). The binding scale was referenced to the aliphatic component of $\mathrm{C} 1 \mathrm{~s}$ spectrum at $284.85 \mathrm{eV}$.

\subsubsection{Nuclear Magnetic Resonance (NMR) spectroscopy}

For ${ }^{1} \mathrm{H}$ NMR analysis, the nanoparticles were dispersed in $\mathrm{D}_{2} \mathrm{O}$, and the spectra were recorded on a Bruker DPX-400 $(400 \mathrm{MHz})$ at 512 scans. Solid-state ${ }^{13} \mathrm{C}$ NMR analysis was performed using an Inova $500 \mathrm{MHz}$ NMR Varian system fitted with a Jacobsen brand $\mathrm{CP} / \mathrm{MAS}$ probe, and 2000 scans were acquired.

\subsubsection{Scanning Electron Microscopy (SEM)}

The shape of the nanoparticles was analyzed by SEM (Quanta 200 FEG, FEI) after gold-sputtering (Gatan 682 Precision and Coating System (PECS)). The average nanoparticle diameters $(\langle D\rangle)$ and their distributions were calculated over 100 particles from SEM images using Image analysis software (NIH, Bethesda, USA). Energy-dispersive X-ray (EDX) mode was used to explore the elemental composition of the material at $30 \mathrm{kV}$ and $4.5 \mathrm{~mA}$ on copper tapes after gold-sputtering.

\subsubsection{Transmission Electron Microscopy (TEM)}

TEM (FEI, Tecnai G2F30) was performed on the nanoparticles. The particles were dispersed in water, and a tiny droplet was dried on a carbon-coated TEM grid. TEM was operated with an accelerating voltage of $300 \mathrm{kV}$. STEM images were captured using a highangle annular dark field (HAADF) detector.

\subsubsection{Brunauer-Emmett-Teller (BET) measurements}

Surface area was measured by a Quantachrome NOVA 2200e series surface analyzer. The adsorption isotherms of nitrogen at $77 \mathrm{~K}$ were investigated using the Brunaur-Emmett-Teller (BET) method in the $P / P_{0}$ range of $0.05-0.3$. The particles were outgassed for $24 \mathrm{~h}$ at $90^{\circ} \mathrm{C}$. The pore size distribution (PSD) was obtained from the adsorption isotherms using the Barrett-Joyner-Halenda (BJH) method.

\subsubsection{UV-Visible spectrophotometer}

The adsorption spectra of the PAHs were gathered on a Cary 100 spectrophotometer. PAH molecules were dissolved in water, and their spectra were collected between 200 and $800 \mathrm{~nm}$ using quartz cuvettes.

\subsection{PAH sorption tests}

Fluorescence spectra of PAH solutions were recorded on a Cary fluorescence spectrophotometer using quartz cuvettes. The excitation wavelengths were calculated from the maximum absorbance observed by UV-spectrum (Fig. S1). Emission wavelength range was from 250 to $600 \mathrm{~nm}$. The fluorescence spectra of PAHs were measured at various concentrations, and the respective calibration curves of the PAHs were plotted (Figs. S2-6 in the Supporting Information). The nanoparticles were treated with PAH molecules for $3 \mathrm{~h}$, and thereafter, they were centrifuged at 10,000 rpm for 10 min. The supernatant part containing free PAHs was measured with a fluorescence spectrophotometer. We also attempted to use cellulose filter membranes to remove the nanoparticles as an alternative approach to the centrifugation, and indeed, we observed that the filter membrane substantially adsorbs PAH molecules. Thus, the nanoparticles were centrifuged to get rid of the nanoparticles from an aqueous phase after exposed to PAHs.

\section{Results and discussion}

Mesostructured silica nanoparticles (MSNs) were synthesized with the formation liquid crystalline phases of cetyltrimethylammonium chloride (CTAC) that served as templates for the in-situ polymerization of tetraethyl orthosilicate (TEOS) in alkaline conditions. This chemistry relies on the hydrolysis of alkoxy groups into silanols and their subsequent condensation reactions to produce $\mathrm{Si}-\mathrm{O}-\mathrm{Si}$ based networks. After the synthesis, MSNs were not harshly treated with chemicals to get rid of all CTAC molecules, but they were just exposed to a mixed solution of acetic acid and methanol. This will of course lead to a MSN-CTAC system rather than a sole MSN particle. As the CTAC moieties in the mesopores is more hydrophobic than the silica and relatively stable in the pores, the total removal of CTAC moieties was not desired for their use for the removal of lipophilic PAH molecules by hydrophobic adsorption. The particles were characterized using SEM, where the spherical nanoparticles with an average nanoparticle size of ca. $100 \mathrm{~nm}$ were observed (Fig. 1(a) and (b)). The mean particle size was measured $\sim 300 \mathrm{~nm}$ in water, suggesting the presence of interconnected particles (Fig. 1(a)-(d)). This structure could be attributed to a low CTAC content. Beyond the interest of this study, monodisperse MSNs can be synthesized with the optimization of formulation parameters.

Cyclodextrin-functionalized silica nanoparticles (CDMSNs) were synthesized using the identical synthesis procedure, but with a further addition of hydroxypropyl- $\beta$-cyclodextrin (HP- $\beta-C D)(23 \%$ $(\mathrm{w} / \mathrm{v})$ of TEOS) or $\beta$-cyclodextrin $(\beta-\mathrm{CD})(150 \%(\mathrm{w} / \mathrm{v})$ of TEOS) prior to the addition of TEOS. Fig. 1(e) and (f) shows spherical hpCDMSNs with a mean particle size at ca. $120 \mathrm{~nm}$. The mean particle diameter of the hpCDMSNs is higher than the pristine MSNs, suggesting that the addition of water-soluble HP- $\beta-C D$ moieties slightly increased the particle size diameter. As the cyclodextrin was added prior to the addition of TEOS, it affected the formation mechanism of the particles, and led to bigger particles. The size distribution of the hpCDMSNs was measured by dynamic light scattering (DLS) showed that the presence of interconnected particles in the range of $150-700 \mathrm{~nm}$, together with a small percentage of single nanoparticles (Fig. $1 \mathrm{~h}$ ). On the other hand, native $\beta-C D$ functionalized MSNs (nCDMSNs) were synthesized by using a substantial amount of $\beta-C D(150 \%$ ( $w / v)$ of TEOS). SEM images of these particle demonstrated the formation of bean-like structure rather than spherical ones (Fig. 1(i) and (j)). Further, these particles with a mean particle size of $\sim 295 \mathrm{~nm}$ and PDI of 0.870 were much larger than the pristine MSNs and hpCDMSNs (Fig. 1(k) and (1)).

The shape and pore structure of the nanoparticles were explored by TEM, where the particles with interconnected structures were observed for both pristine MSNs and hpCDMSNs (Fig. 2). High-resolution TEM (HRTEM) image of the pristine MSNs revealed a periodic pore structure, suggesting a mesoporous network architecture within the particles. The physical incorporation of cyclodextrins affected the particle shape and pore size, particularly for the $\beta-C D$ functionalized particles. SEM analysis demonstrated a slight increase in the particle size by $20 \%$ (from ca. 100 to $120 \mathrm{~nm}$ ) for the hpCDMSNs. The mean pore size for the pristine MSNs was found as $2.2 \mathrm{~nm}$ by TEM, while it was measured as 

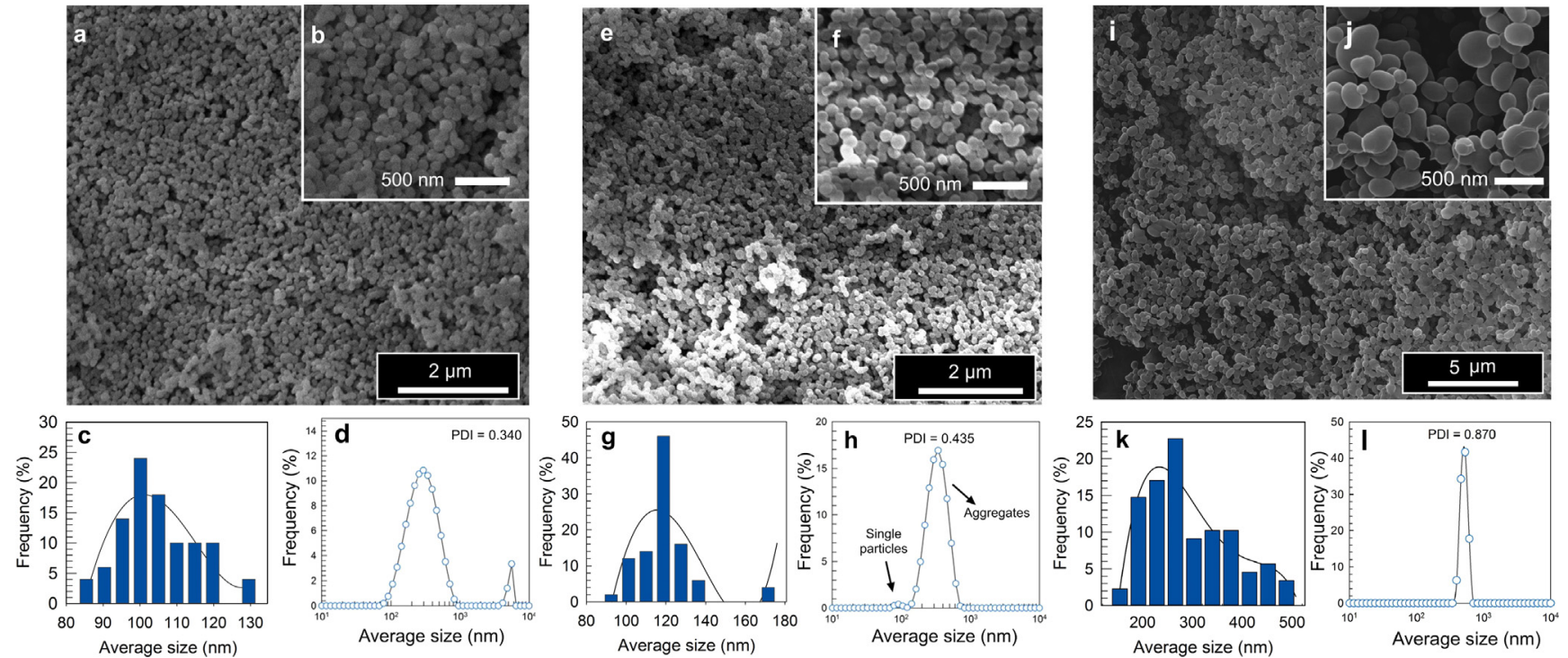

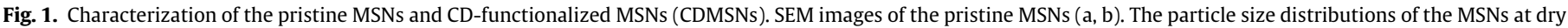

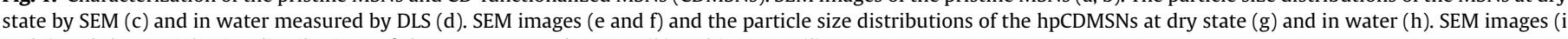
and $\mathrm{j}$ ) and the particle size distributions of the nCDMSNs at dry state (k) and in water (1).

$2.7 \mathrm{~nm}$ for the hpCDMSNs, suggesting that the addition of HP- $\beta-\mathrm{CD}$ led to larger pores. However, TEM images of the nCDMSNs showed obvious changes on the particles, not only the size of particles, but also pore structure. Almost a threefold rise in particle size was observed, and the pore size decreased to $0.97 \mathrm{~nm}$, suggesting a microporous structure rather than a mesoporous one. By taking account of the molecular size of $\beta-C D(\sim 1.6 \mathrm{~nm})$, it was obvious that the particle surface was decorated by cyclodextrin moieties. The specific surface areas of pristine MSNs, hpCDMSNs and nCDMSNs were found as $232.49,142.47$ and $61.56 \mathrm{~m}^{2} / \mathrm{g}$, respectively. The decrease in surface areas for CDMSNs samples could be attributed to the presence of CTAC and CDs inside the pores that reduce surface areas. Nevertheless, CDMSNs have shown high scavenging capacities for the removal of PAH molecules due to the presence of $\mathrm{CD}$ moieties as discussed in the following sections.

After heat treatment at $450{ }^{\circ} \mathrm{C}$ for $2 \mathrm{~h}$, the weight losses for the hpCDMSNs and pristine MSNs were respectively found as $51 \%$ and $38.5 \%$. Generally, an initial weight loss ( $2 \%)$ on the particle weight during the heating process could be attributed to the release of water molecules, while the rest was due to the pyrolysis of CTAC surfactant and CD moieties on the silica network. However, after the removal of cyclodextrins and CTAC moieties, TGA analysis of the hpCDMSNs revealed a small loss on the particle weight due to the pyrolysis of cyclodextrins above $300^{\circ} \mathrm{C}$ (Fig. S7). HR-TEM and STEM analyses after the heat treatment at $450^{\circ} \mathrm{C}$ for $2 \mathrm{~h}$ showed the presence of a honeycomb-type mesoporous structure for both hpCDMSNs and pristine MSNs (Figs. S8 and 9). After heat treatment, both nanoparticles preserved interconnected particle structure, while the particles formed by $\beta$-CDs were in the shape of single particles.

Cyclodextrin-functionalized MSNs before and after the removal of CTAC moieties were analyzed by FT-IR (Fig. S10), where typical peaks assigned to a $\mathrm{C}-\mathrm{H}$ stretching bond of the CTAC moieties could be seen at 2860 and $2930 \mathrm{~cm}^{-1}$, indicating the formation of MSN-CTAC. After the removal of the CTAC and CD moieties, a significant decrease in the corresponding peaks was observed. The bending vibration of $\mathrm{Si}-\mathrm{O}-\mathrm{Si}$ was observed at $\sim 460 \mathrm{~cm}^{-1}$, while the peak appeared at $802 \mathrm{~cm}^{-1}$ was assigned to the symmetric stretching vibration of $\mathrm{Si}-\mathrm{OH}$. Normally, the characteristic absorption bands of cyclodextrins appear at $\sim 1030,1080$ and $1155 \mathrm{~cm}^{-1}$ for $\mathrm{C}-\mathrm{C}$ and $\mathrm{C}-\mathrm{O}$ stretching vibrations and the asymmetric stretching of $\mathrm{C}-\mathrm{O}-\mathrm{C}$ glycosidic bonds. However, the bands between 1050 and $1200 \mathrm{~cm}^{-1}$ related to the various $\mathrm{C}-\mathrm{O}$ stretching vibrations of the cyclodextrins were overlapped with a strong and broad $\mathrm{Si}-\mathrm{O}-\mathrm{Si}$ asymmetric stretching vibration of the MSNs appeared at $\sim 1100 \mathrm{~cm}^{-1}$. However, it has been widened in the case of $\mathrm{SiO}_{2}$-cyclodextrin hybrids due to the overlapping of $\mathrm{Si}-\mathrm{O}-\mathrm{Si}$, $\mathrm{Si}-\mathrm{O}-\mathrm{C}$, and $\mathrm{C}-\mathrm{O}$ stretching peaks. It was previously reported that in organo-silica hybrid networks, an asymmetric stretching vibration of $\mathrm{Si}-\mathrm{O}-\mathrm{Si}$ splits into two separate bands, suggesting the formation of a hybrid matrix [46]. Even after the removal of CTAC moieties, hpCDMSNs show a characteristics $\mathrm{C}-\mathrm{H}$ vibration peak of the HP- $\beta-C D$ at $2930 \mathrm{~cm}^{-1}$, suggesting CD attachment at some extent. On the other hand, nCDMSNs show all characteristic peaks of $\beta$-CD (i.e. 942, 1094, 1166, 1337, 2929 and $3467 \mathrm{~cm}^{-1}$ ) and as well as $\mathrm{Si}-\mathrm{O}-\mathrm{Si}$ peaks of the silica network, and thus demonstrate the formation of a hybrid CDMSN nanonetwork with high cyclodextrin loading (see Fig. S10).

For the confirmation of the adsorption of cyclodextrins on the particle surface, XPS was used to elucidate the atomic composition (Fig. 3), and the corresponding compositional data were given in Table S1 (see Supporting Information). With CD functionalization, the proportion of silicon (Si2s and Si2p) declined from 35.34 to $28.42 \%$ while the carbon content increased from 32 to $37 \%$. Whereas, for the nCDMSNs, C and Si contents were $58 \%$ and $20 \%$, respectively, suggesting a substantial amount of the incorporated CDs. Since cyclodextrins are compositionally made of carbon (C) and oxygen $(\mathrm{O})$, a rise in $\mathrm{C}$ content in the atomic composition of particles could be attributed to the presence of the cyclodextrin and CTAC on the surface. Further, ${ }^{1} \mathrm{H}$ NMR analysis of the CDMSNs in $\mathrm{D}_{2} \mathrm{O}$ shows the characteristic peaks of cyclodextrin-backbone protons between 3 and $6 \mathrm{ppm}$, together with the methyl protons of hydroxypropyl moieties at $1.1 \mathrm{ppm}$ (Fig. S11 in the Supporting Information). The methylene protons adjacent to $\mathrm{Si}\left(\mathrm{Si}-\mathrm{CH}_{2}\right)$ were detected at $\sim 0.56 \mathrm{ppm}$, suggesting a silica-cyclodextrin hybrid network. However, after the removal of adsorbed CTAC and CD moieties, almost all cyclodextrins and CTAC moieties were detached from the particles, and very small peaks related to cyclodextrins could be detected in the respective region (Fig. S11, inset). ${ }^{1} \mathrm{H}$ NMR spectrum of the nCDMSNs clearly shows all protons peaks 
(a)
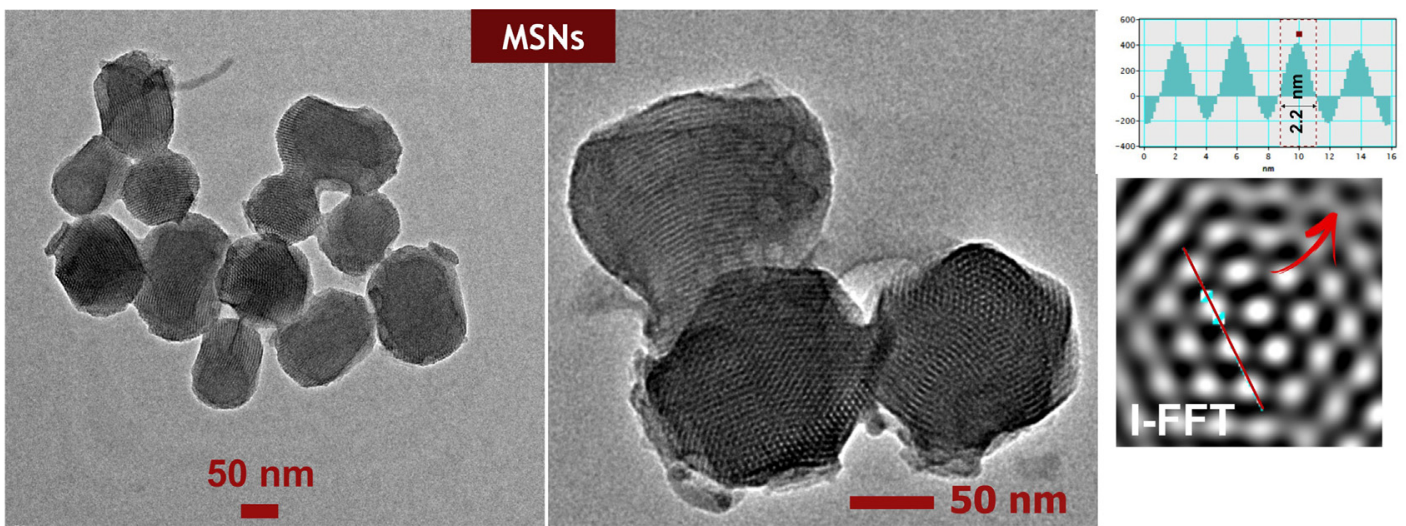

(b)
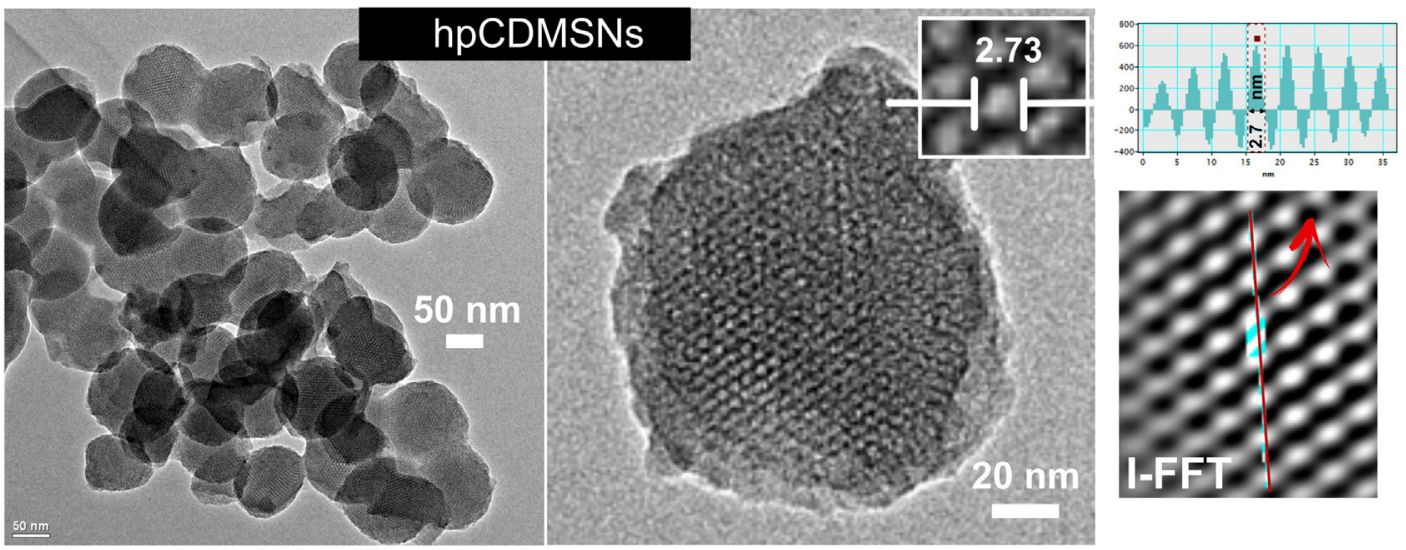

(c)
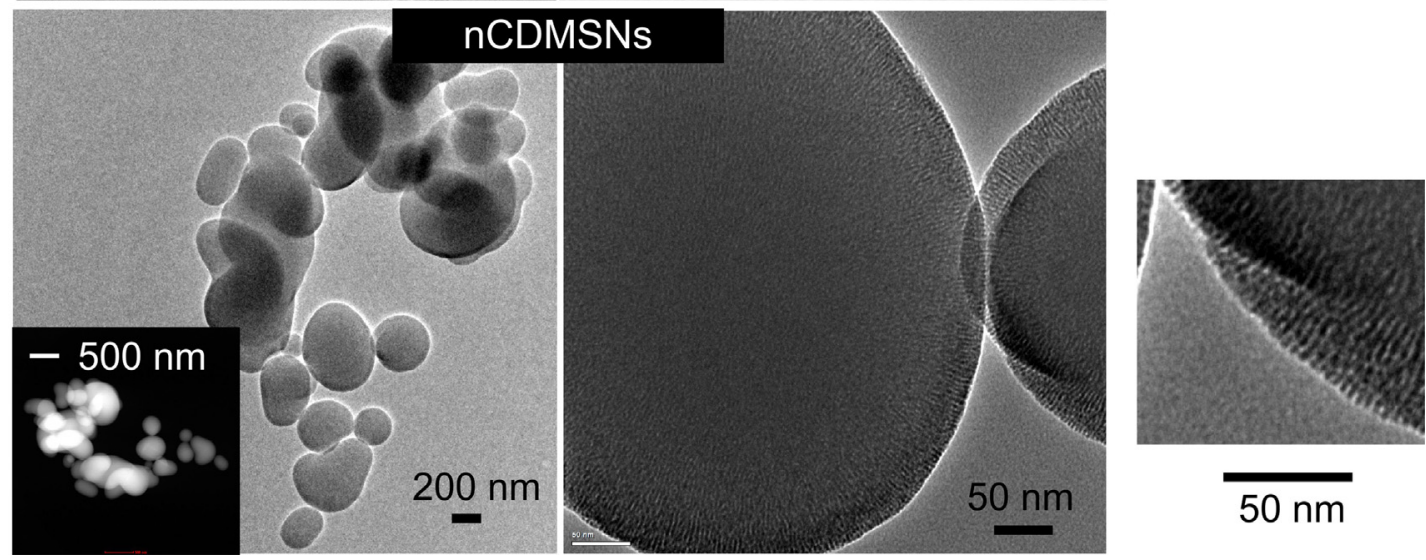

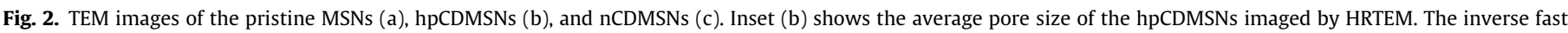

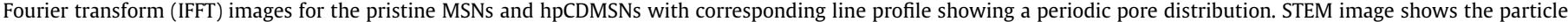
structure of the nCDMSNs.

of adsorbed $\beta$-CDs (Fig. S12). Likewise, TGA analysis of the hpCDMSNs revealed a slight weight loss between 300 and $350{ }^{\circ} \mathrm{C}$ because of the adsorbed cyclodextrins (Fig. S7). In contrast, $\beta-\mathrm{CD}$ functionalized MSNs showed a significant weight loss ( 40 wt\%) due to the incorporated CDs. The pyrolysis of the $\beta-C D$ took place at ca. $320^{\circ} \mathrm{C}$, while very small weight loss was observed for CTAC moieties around $280^{\circ} \mathrm{C}$. For further confirmation of the adsorption of cyclodextrins, solid-state ${ }^{13} \mathrm{C}$ NMR analysis was performed on the particles, where the characteristic peaks of the $\beta-C D$ were appeared in the ranges of $97-106 \mathrm{ppm}$ for $\mathrm{C}-1$, and $78-86 \mathrm{ppm}$ for $\mathrm{C}-2,3,4,5$ (Fig. S16).

The presence of cyclodextrins on the particle surface could be seen over the deconvoluted C1s spectra of both nanoparticles (pristine MSNs and hpCDMSNs) (Fig. 3(b) and (c)). The peak appeared at $284.85 \mathrm{eV}$ is considered as an aliphatic carbon ( $\mathrm{C}-\mathrm{C}$ or $\mathrm{C}-\mathrm{H})$, while the peak at $286.5 \mathrm{eV}$ corresponds to the carbon bound to oxygen (C-O). Since the cyclodextrins have high number of $\mathrm{C}-\mathrm{O}$ bonds, a rise in the peak at $286.5 \mathrm{eV}$ can be assigned to the incorporated cyclodextrins. Furthermore, the peak at $287.5 \mathrm{eV}$ suggests the presence of carbon-oxygen-carbon $(\mathrm{C}-\mathrm{O}-\mathrm{C})$ bond of cyclodextrins and the carbon-nitrogen $(\mathrm{C}-\mathrm{N})$ bond of CTAC. Fig. 3(b) and (c) shows the $\mathrm{C} 1 \mathrm{~s}$ narrow spectra of both nanoparticles (hpCDMSNs and pristine MSNs). Similarly, energy-dispersive X-ray spectroscopy (EDX) analysis on both particles showed larger oxygen (O) peak for hpCDMSNs compared to the pristine MSNs due to cyclodextrin moieties (Fig. S13). Similarly, the carbon peak was more distinctive for hpCDMSNs. Both MSN and hpCDMSN samples through XPS and EDX suggest the presence of cyclodextrins on the particles.

To figure out the cyclodextrin localization, the zeta-potential analysis was performed. This analysis method allows qualitative 

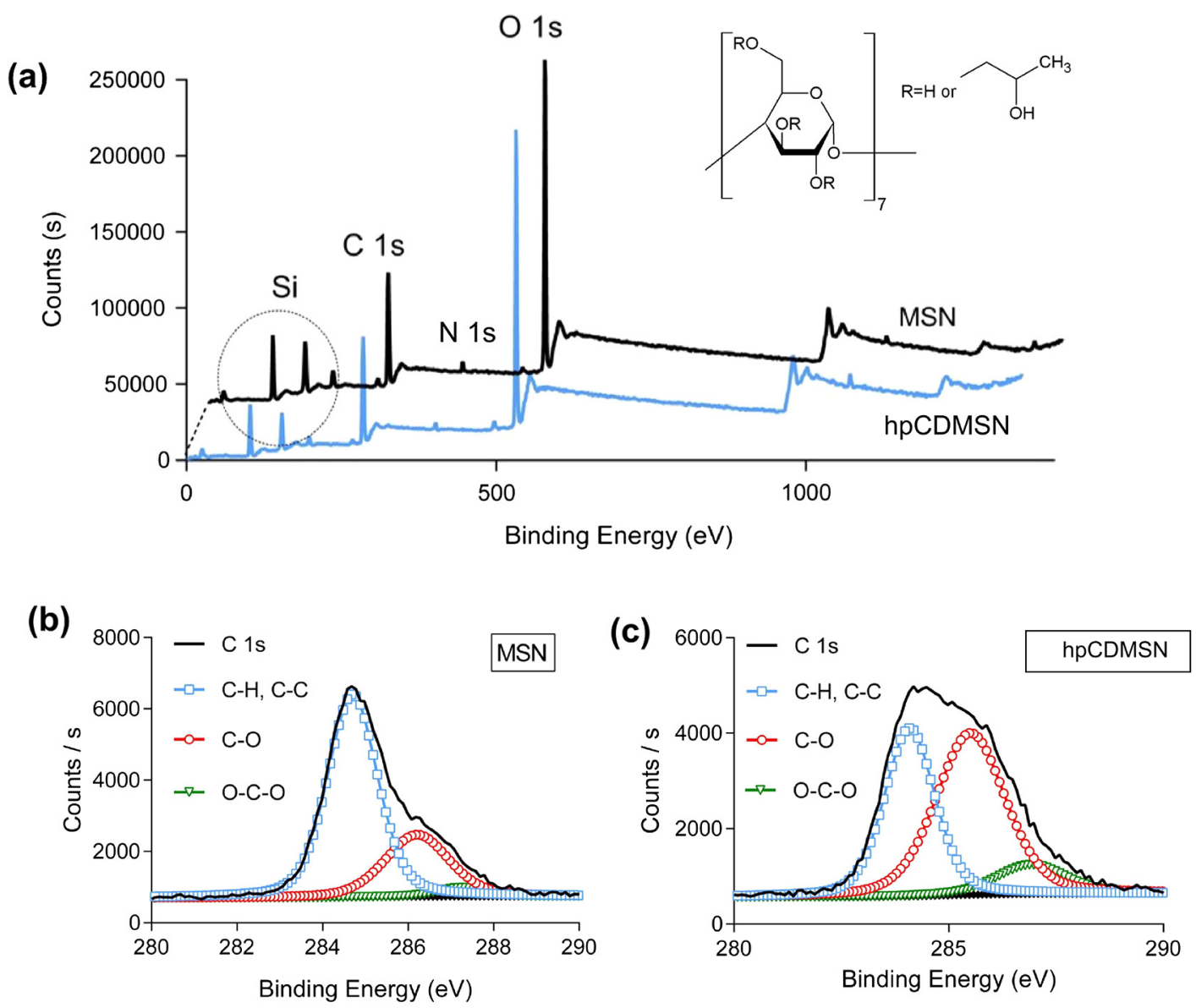

Fig. 3. XPS survey spectra (a) and the deconvoluted C1s spectra (b and c) of the pristine MSNs and hpCDMSNs.

identification of cyclodextrins at the slipping plane located just outer surface of the particles. Fig. S14 shows the pH-dependent zeta-potentials of the silica nanoparticles in the $\mathrm{pH}$ range of 3-12. At high $\mathrm{pH}$ (basic aqueous values), CDMSNs and pristine MSNs have similar zeta $(\zeta)$ potentials at ca. $-40 \mathrm{mV}$. While lowering the $\mathrm{pH}$ just below 6 , the $\zeta$ is varied depending on the presence or absence of cyclodextrin. The hpCDMSNs have zeta-potential between 40 and $45 \mathrm{mV}$, while the pristine MSNs have zetapotentials between 20 and $25 \mathrm{mV}$ in the identical $\mathrm{pH}$ range. This significant difference in the zeta potentials between these two nanoparticles suggests that the CDMSNs have a considerable amount of cyclodextrins on the particle surface so that the hydro$\mathrm{xyl}$ groups become protonated at acidic $\mathrm{pH}$, yielding positive zeta potentials.

The structural analyses of nanoparticles were performed by WAXD, and the respective XRD patterns of nanoparticles are shown in Fig. S15, where pristine MSNs and hpCDMSNs gave a broad peak centered at $23^{\circ}$ due to amorphous $\mathrm{SiO}_{2}$ [47]. The silica nanoparticles are amorphous materials and do not show sharp crystalline peaks, with only exception of the peaks of periodic pores that appear in the range of $2-8^{\circ}$. Further, HP- $\beta$-CD has also an amorphous structure. The pristine MSNs show typical diffraction peaks (100), (110) and (200) of the highly ordered hexagonal pore structure, while smaller peaks were observed for the hpCDMSNs, suggesting less-ordered mesopores. This is could be ascribed that the incorporation of cyclodextrins affected the pore-architecture, but the sample preserved a mesoporous network as confirmed by TEM analyses. In contrast, the nCDMSNs exhibited a very different XRD pattern, where the peaks related to the periodic pores appeared in the range $2-10^{\circ}$, and the crystalline peaks of $\beta$-CDs were observed in the range of $10-50^{\circ}$, suggesting that surface decoration of the particles with $C D$ moieties was successful.

In this study, the PAH sorption tests were performed by treating silica particles with several PAHs under continuous stirring (Fig. 4). Afterward, the nanoparticles were removed by the centrifugation at $13,000 \mathrm{rpm}$ for $10 \mathrm{~min}$, and the supernatant part was taken into vials and measured by a fluorescence spectrophotometer at excitation wavelengths determined by UV/Vis analyses (Fig. S1, Supporting Information). Fluorescence spectra of all PAH solutions revealed significant reductions in emission spectra upon treatment with nanoparticles (Fig. 4). Even though HP- $\beta-C D$ functionalized MSNs exhibited slightly better PAH sorption capacities up to $21 \%$, there was no vast difference in the $\mathrm{PAH}$ removal capacities between hpCDMSNs and pristine MSNs due to the low CD loadings. On the other hand, $\beta$-CD functionalized MSNs, which were synthesized at very high $C D$ content, showed the highest reduction in the fluorescence spectrum of the respective PAH molecule after having treated for $3 \mathrm{~h}$. Even though the surface area of the pristine MSN is much higher than the CD-functionalized MSNs, the presence of high CD content, particularly for the nCDMSNs, allows the efficient removal of the PAH molecules. Moreover, the performance of such particles was comparable to that of activated carbon, which demonstrated the complete removal of PAH molecules (Fig. 4).

The overall PAH-removal performance of the CDMSNs and pristine MSNs was shown in Fig. 5, where the scavenging capacities of the particles were expressed as mg PAH adsorbed on the gram silica particles. The PAH sorption capacities of the nanoparticles were found over $0.3 \mathrm{mg}$, even reaching to $1.65 \mathrm{mg}$ with a mean sorption capacity higher than $1 \mathrm{mg}$, suggesting that the nanoparticles are substantially good in snaring of toxic PAH compounds from aqueous solutions. The nCDMSNs showed the highest scavenging 

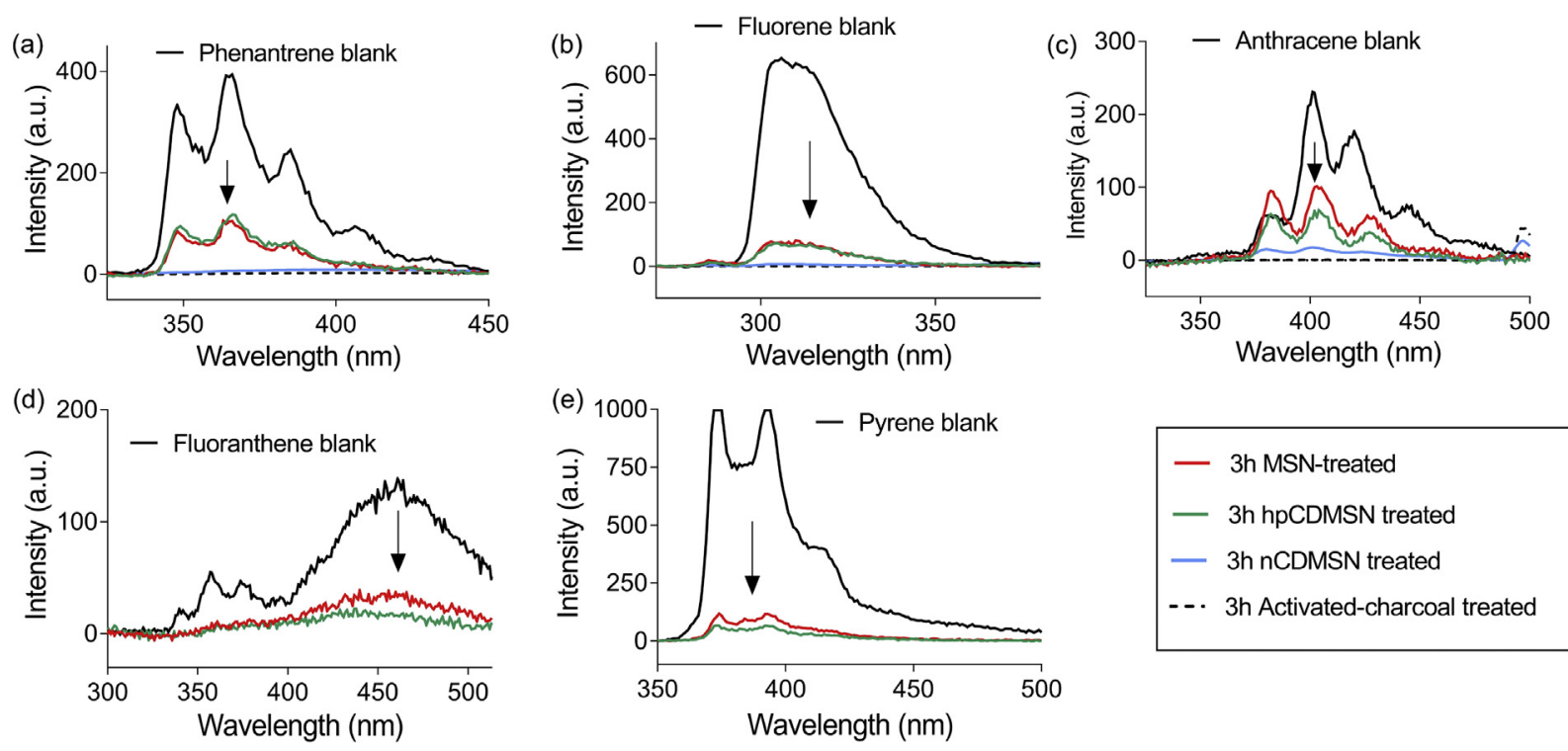

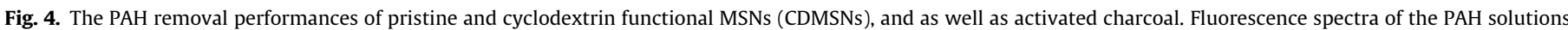
((a) phenanthrene, (b) fluorene, (c) anthracene, (d) fluoranthene and (e) pyrene before and after treatments with the nanoparticles.
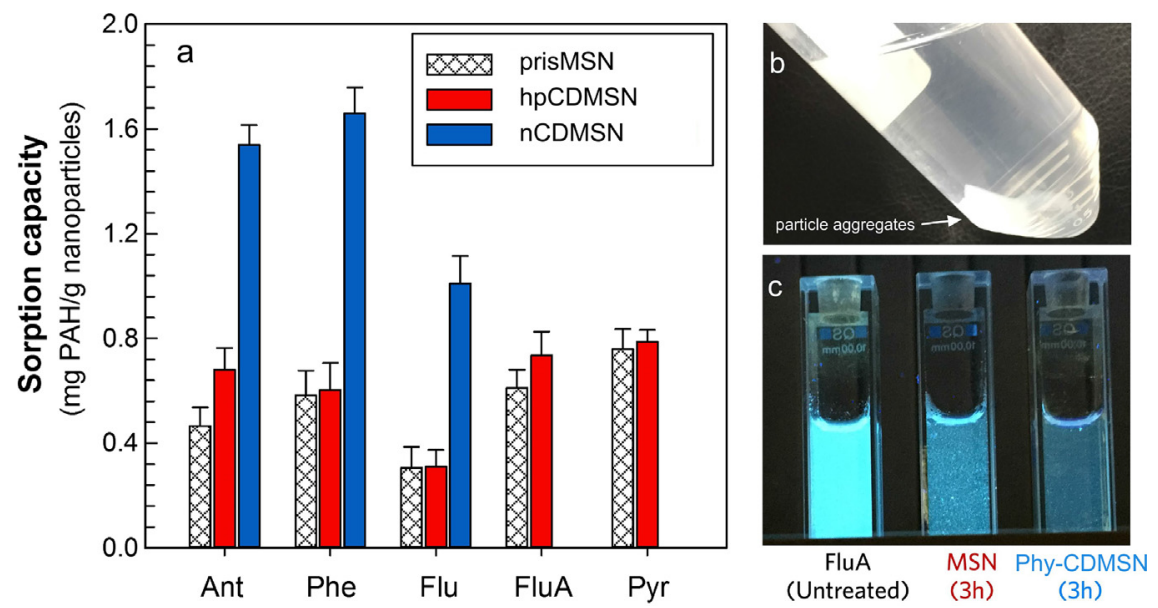

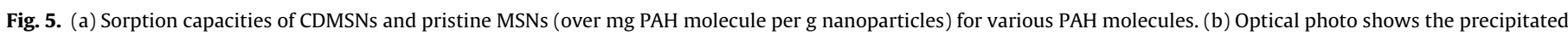

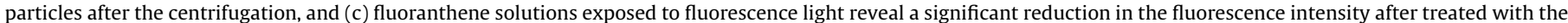
hPCDMSNs.

capacity to those pristine MSNs and hpCDMSNs with a $\sim 2$-fold rise in the sorption capacity of PAHs. This high sorption capacity was attributed to high CD loading for nCDMSNs. Overall, the CDMSNs were very good in the removal of toxic PAHs, and thereby have high potential as sorbent platforms in PAH removal from aquatic environments. The incorporation of $\mathrm{CD}$ motifs on the nanomaterials is a very important parameter for their sorption applications and enhances PAH removal performance of the materials.

\section{Conclusion}

Cyclodextrin-functionalized mesostructured silica nanoparticles (CDMSNs) were generated in a simple, one-pot reaction system, and exploited for the removal of genotoxic polycyclic aromatic hydrocarbons (PAHs) from aqueous solutions. Surfactanttemplated, base-catalyzed MSNs were successfully synthesized in the presence and absence of cyclodextrins, and several techniques (SEM, TEM, DLS, XPS, FTIR, Zeta potential and BET) were used to characterize the nanoparticles. TGA and solid-state ${ }^{13} \mathrm{C} N M R$ analyses confirmed the presence of adsorbed cyclodextrin moieties, while HR-TEM revealed periodic porous structures. Even though the pristine MSNs exhibited very high sorption capacities due to their highly porous structures, which mimicked as if nanomembranes once the polluted water passes through pores, cyclodextrin functionalization significantly boosted the sorption capacity of the nanoparticles by a twofold of the pristine MSNs. This one-pot fabrication pathway of cyclodextrin-functionalized silica nanoparticles (CDMSNs) seems a promising approach with their high scavenging capacities for the removal of genotoxic PAH molecules from aquatic environments with performances comparable to that of activated charcoal. The chemical modification of such functional motifs onto silica particles and their recyclable use in PAH removal is currently under investigation.

\section{Acknowledgements}

T. U. acknowledges The Turkish Academy of Sciences - Outstanding Young Scientists Award Program (TUBA-GEBIP)-Turkey 
for partial funding of the research. F. T. thanks to the Tubitak Co-Funded Brain Circulation Scheme (Project No: 116C031).

\section{Appendix A. Supplementary material}

Supplementary data associated with this article can be found, in the online version, at http://dx.doi.org/10.1016/j.jcis.2017.03.015.

\section{References}

[1] V.J. Melendez-Colon, A. Luch, A. Seidel, W.M. Baird, Cancer initiation by polycyclic aromatic hydrocarbons results from formation of stable DNA adducts rather than apurinic sites, Carcinogenesis 20 (10) (1999) 1885-1891.

[2] J. van Grevenynghe, M. Bernard, S. Langouet, C. Le Berre, T. Fest, O. Fardel, Human CD34-positive hematopoietic stem cells constitute targets for carcinogenic polycyclic aromatic hydrocarbons, J. Pharmacol. Exp. Therap. 314 (2) (2005) 693-702.

[3] T. Shimada, Y. Fujii-Kuriyama, Metabolic activation of polycyclic aromatic hydrocarbons to carcinogens by cytochromes P450 1A1 and1B1, Cancer Sci. 95 (1) (2004) 1-6.

[4] L.K. Siddens, K.L. Bunde, T.A. Harper Jr, T.J. McQuistan, C.V. Löhr, L.M. Bramer, K. M. Waters, S.C. Tilton, S.K. Krueger, D.E. Williams, W.M. Baird, Cytochrome P450 1b1 in polycyclic aromatic hydrocarbon (PAH)-induced skin carcinogenesis: tumorigenicity of individual PAHs and coal-tar extract, DNA adduction and expression of select genes in the Cyp1b1 knockout mouse, Toxicol. Appl. Pharmacol. 287 (2) (2015) 149-160.

[5] F.J. Van Schooten, A.W. Boots, A.M. Knaapen, R.W.L. Godschalk, L.M. Maas, P.J.A Borm, M. Drent, J.A. Jacobs, Myeloperoxidase (MPO)-463G -> a reduces MPO activity and DNA adduct levels in bronchoalveolar lavages of smokers, Cancer Epidemiol. Biomarkers Prevent. 13 (5) (2004) 828-833.

[6] O. Barbieri, E. Ognio, O. Rossi, S. Astigiano, L. Rossi, Embryotoxicity of benzo(a) pyrene and some of its synthetic derivatives in Swiss mice, Cancer Res. 46 (1) (1986) 94-98.

[7] C. Legraverend, T.M. Guenthner, D.W. Nebert, Importance of the route of administration for genetic differences in benzo[a]pyrene-induced in utero toxicity and teratogenicity, Teratology 29 (1) (1984) 35-47.

[8] Environmental Protection Agency, Polycyclic Aromatic Hydrocarbons (PAHs), EPA, US, 2008, p. 2008.

[9] K.-H. Kim, S.A. Jahan, E. Kabir, R.J.C. Brown, A review of airborne polycyclic aromatic hydrocarbons (PAHs) and their human health effects, Environ. Int. 60 (2013) 71-80.

[10] K. Srogi, Monitoring of environmental exposure to polycyclic aromatic hydrocarbons: a review, Environ. Chem. Lett. 5 (4) (2007) 169-195.

[11] A.K. Haritash, C.P. Kaushik, Biodegradation aspects of Polycyclic Aromatic Hydrocarbons (PAHs): a review, J. Hazard. Mater. 169 (1-3) (2009) 1-15.

[12] R.E. Laflamme, R.A. Hites, The global distribution of polycyclic aromatic hydrocarbons in recent sediments, Geochim. Cosmochim. Acta 42 (3) (1978) 289-303.

[13] C.A. Menzie, B.B. Potocki, J. Santodonato, Exposure to carcinogenic PAHs in the environment, Environ. Sci. Technol. 26 (7) (1992) 1278-1284.

[14] G.P. Pfeifer, M.F. Denissenko, M. Olivier, N. Tretyakova, S.S. Hecht, P. Hainaut, Tobacco smoke carcinogens, DNA damage and p53 mutations in smokingassociated cancers, Oncogene 21 (48) (2002) 7435-7451.

[15] J.C. Barnes, M. Juríček, N.L. Strutt, M. Frasconi, S. Sampath, M.A. Giesener, P.L. McGrier, C.J. Bruns, C.L. Stern, A.A. Sarjeant, J.F. Stoddart, ExBox: a polycyclic aromatic hydrocarbon scavenger, J. Am. Chem. Soc. 135 (1) (2013) 183-192.

[16] C. Viglianti, K. Hanna, C. de Brauer, P. Germain, Removal of polycyclic aromatic hydrocarbons from aged-contaminated soil using cyclodextrins: experimental study, Environ. Pollut. 140 (3) (2006) 427-435.

[17] H. Li, F. Qu, Selective inclusion of polycyclic aromatic hydrocarbons (PAHs) on calixarene coated silica nanospheres englobed with CdTe nanocrystals, J. Mater. Chem. 17 (33) (2007) 3536-3544.

[18] C. Peinador, E. Pía, V. Blanco, M.D. García, J.M. Quintela, Complexation of pyrene in aqueous solution with a self-assembled palladium metallocycle, Org. Lett. 12 (7) (2010) 1380-1383.

[19] F. Brandl, N. Bertrand, E.M. Lima, R. Langer, Nanoparticles with photoinduced precipitation for the extraction of pollutants from water and soil, Nat. Commun. 6 (2015) 7765.

[20] I.I. Slowing, J.L. Vivero-Escoto, C.-W. Wu, V.S.Y. Lin, Mesoporous silica nanoparticles as controlled release drug delivery and gene transfection carriers, Adv. Drug Deliv. Rev. 60 (11) (2008) 1278-1288.

[21] F. Tang, L. Li, D. Chen, Mesoporous silica nanoparticles: synthesis, biocompatibility and drug delivery, Adv. Mater. 24 (12) (2012) 1504-1534.

[22] S.-H. Wu, C.-Y. Mou, H.-P. Lin, Synthesis of mesoporous silica nanoparticles, Chem. Soc. Rev. 42 (9) (2013) 3862-3875.
[23] T. Asefa, Z. Tao, Biocompatibility of mesoporous silica nanoparticles, Chem. Res. Toxicol. 25 (11) (2012) 2265-2284.

[24] F. Torney, B.G. Trewyn, V.S.Y. Lin, K. Wang, Mesoporous silica nanoparticles deliver DNA and chemicals into plants, Nat. Nano 2 (5) (2007) 295-300.

[25] A. Popat, J. Liu, Q. Hu, M. Kennedy, B. Peters, G.Q. Lu, S.Z. Qiao, Adsorption and release of biocides with mesoporous silica nanoparticles, Nanoscale 4 (3) (2012) 970-975.

[26] A. Popat, S.B. Hartono, F. Stahr, J. Liu, S.Z. Qiao, G. Qing Lu, Mesoporous silica nanoparticles for bioadsorption, enzyme immobilisation, and delivery carriers, Nanoscale 3 (7) (2011) 2801-2818.

[27] X. Li, J. Zhang, H. Gu, Adsorption and desorption behaviors of DNA with magnetic mesoporous silica nanoparticles, Langmuir 27 (10) (2011) 60996106.

[28] E. Morillo, M.A. Sánchez-Trujillo, J.R. Moyano, J. Villaverde, M.E. GómezPantoja, J.I. Pérez-Martínez, Enhanced solubilisation of six PAHS by three synthetic cyclodextrins for remediation applications: molecular modelling of the inclusion complexes, PLoS ONE 7 (9) (2012) e44137.

[29] G. Shixiang, W. Liansheng, H. Qingguo, H. Sukui, Solubilization of polycyclic aromatic hydrocarbons by $\beta$-cyclodextrin and carboxymethyl- $\beta$-cyclodextrin, Chemosphere 37 (7) (1998) 1299-1305.

[30] N.-D. Estrella, G.-H. José Antonio, Cyclodextrins, Encyclopedia of Biotechnology in Agriculture and Food, Taylor \& Francis, 2010, pp. 187-190.

[31] G. Crini, Review: a history of cyclodextrins, Chem. Rev. 114 (21) (2014) 1094010975.

[32] B. Voncina, V. Vivod, Cyclodextrins in textile, Cyclodextrins in textile finishing, 2013.

[33] A. Celebioglu, F. Kayaci, S.I. Kusku, E. Durgun, T. Uyar, Polymer-free nanofibers from vanillin/cyclodextrin inclusion complexes: high thermal stability, enhanced solubility and antioxidant property, Food Funct. 7 (2016) 31413153.

[34] T. Uyar, R. Havelund, Y. Nur, J. Hacaloglu, F. Besenbacher, P. Kingshott, Molecular filters based on cyclodextrin functionalized electrospun fibers, J. Membr. Sci. 332 (1-2) (2009) 129-137.

[35] T. Uyar, R. Havelund, J. Hacaloglu, F. Besenbacher, P. Kingshott, Functional electrospun polystyrene nanofibers incorporating $\alpha-, \beta-$, and $\gamma$-cyclodextrins: comparison of molecular filter performance, ACS Nano 4 (9) (2010) 5121 5130.

[36] T. Uyar, R. Havelund, Y. Nur, A. Balan, J. Hacaloglu, L. Toppare, F. Besenbacher, P. Kingshott, Cyclodextrin functionalized poly(methyl methacrylate) (PMMA) electrospun nanofibers for organic vapors waste treatment, J. Membr. Sci. 365 (1-2) (2010) 409-417.

[37] F. Kayaci, T. Uyar, Electrospun polyester/cyclodextrin nanofibers for entrapment of volatile organic compounds, Polym. Eng. Sci. 54 (12) (2014) $2970-2978$.

[38] A. Celebioglu, H.S. Sen, E. Durgun, T. Uyar, Molecular entrapment of volatile organic compounds (VOCs) by electrospun cyclodextrin nanofibers, Chemosphere 144 (2016) 736-744.

[39] A. Celebioglu, T. Uyar, Electrospun gamma-cyclodextrin ([gamma]-CD) nanofibers for the entrapment of volatile organic compounds, RSC Adv. 3 (45) (2013) 22891-22895.

[40] F. Kayaci, H.S. Sen, E. Durgun, T. Uyar, Electrospun nylon 6,6 nanofibers functionalized with cyclodextrins for removal of toluene vapor, J. Appl. Polym. Sci. 132 (18) (2015) 41941.

[41] A. Celebioglu, S. Demirci, T. Uyar, Cyclodextrin-grafted electrospun cellulose acetate nanofibers via "Click" reaction for removal of phenanthrene, Appl. Surf. Sci. 305 (2014) 581-588.

[42] F. Kayaci, Z. Aytac, T. Uyar, Surface modification of electrospun polyester nanofibers with cyclodextrin polymer for the removal of phenanthrene from aqueous solution, J. Hazard. Mater. 261 (2013) 286-294.

[43] K. Yang, B. Chen, L. Zhu, Graphene-coated materials using silica particles as a framework for highly efficient removal of aromatic pollutants in water, Sci. Rep. 5 (2015) 11641.

[44] P. Laveille, A. Falcimaigne, F. Chamouleau, G. Renard, J. Drone, F. Fajula, S. Pulvin, D. Thomas, C. Bailly, A. Galarneau, Hemoglobin immobilized on mesoporous silica as effective material for the removal of polycyclic aromatic hydrocarbons pollutants from water, New J. Chem. 34 (10) (2010) 2153-2165.

[45] S. Hall, R. Tang, J. Baeyens, R. Dewil, Removing polycyclic aromatic hydrocarbons from water by adsorption on silicagel, Polycyclic Aromatic Comp. 29 (3) (2009) 160-183.

[46] S. Smitha, P. Shajesh, P. Mukundan, K.G.K. Warrier, Sol-gel synthesis of biocompatible silica-chitosan hybrids and hydrophobic coatings, J. Mater. Res. 23 (8) (2008) 2053-2060.

[47] H.-X. Guo, X.-P. Zhao, H.-L. Guo, Q. Zhao, Preparation of porous $\mathrm{SiO}_{2} / \mathrm{Ni} / \mathrm{TiO}_{2}$ multicoated microspheres responsive to electric and magnetic fields, Langmuir 19 (23) (2003) 9799-9803. 